\title{
Experimental evaluation of Resonant Tunnelling Diode Oscillators employing Advanced Modulation Formats
}

\author{
J. S. Tavares ${ }^{1,2}$, L. M. Pessoa ${ }^{1}$, H. M. Salgado ${ }^{1,2}$ \\ ${ }^{1}$ INESC TEC, Portugal \\ ${ }^{2}$ Faculty of Engineering, University of Porto, Portugal \\ e-mail: joana.tavares@fe.up.pt
}

\begin{abstract}
The performance of Resonant Tunnelling Diode (RTD) oscillators with an optical window is evaluated experimentally, in the transmission of advanced modulation formats using electrical and optical modulation, for the first time. Additionally, the impact of phase noise in the transmission performance is also assessed.
\end{abstract}

Keywords: advanced modulation formats, optical modulation, oscillator, phase noise, resonant tunnelling diode (RTD).

\section{INTRODUCTION}

RTD devices with photo-detection capabilities can be achieved by including a photo-conductive layer within the Double barrier quantum well (DBQW) epi-layer structure of standard RTDs [1], and providing access to this layer through an optical window on the top of the device mesa. These devices have been shown to provide high responsivities, high gain-bandwidth efficiency products, low switching energies and low voltage operation characteristics [2]. RTD-PDs were shown to be suitable for fibre-wireless interfaces, by converting either a baseband modulated or RF modulated subcarrier optical signal, impinging on its optical window, to a high frequency RF carrier [3]. Previously, we experimentally evaluated the optical response of the first RTD photodetector (RTD-PD) oscillators available from the iBROW project and demonstrated the feasibility of amplitude and frequency modulation by light injection [4].

In this work, we evaluate experimentally, for the first time, the performance of RTD-PD oscillators in the transmission of advanced modulation formats using electrical and optical modulation. Moreover, the impact arising from the RTD phase noise is evaluated by means of locking the RTD oscillation carrier. Phase noise is a main limiting factor in oscillators and consists of random rotations of the signal constellation that originates detection errors [5].

The organization of this paper is as follows. Section 2 describes the experimental setups implemented to assess the performance of the RTD-PDs using electrical and optical modulation. Section 3 presents the experimental results and discussion. Section 4 concludes the paper.

\section{EXPERIMENTAL SETUP}

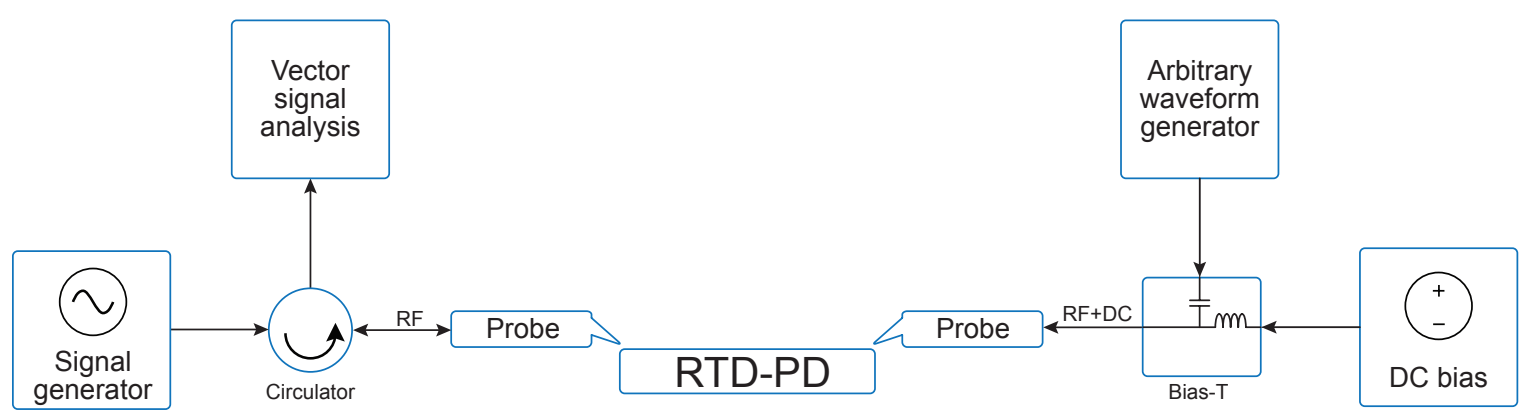

Figure 1. Experimental setup for electrical modulation.

Figure 1 shows the experimental setup used for the transmission of advanced modulation formats. The complex modulations are generated by an Arbitrary Waveform Generator (AWG) that sends a subcarrier modulated complex signal to the DC port of the RTD through a bias T. On the RF port of the RTD, a spectrum analyser is connected, that runs a Vector Signal Analysis (VSA) software, used for evaluating the quality of the transmitted signal. A signal generator is also connected through a circulator on the RF port, that allows the locking of the RTD oscillation carrier and its impact on phase noise is evaluated. The RTD is biased in the NDR (negative differential resistance) which guarantees oscillation. Injection of a sinewave close to the oscillation frequency of the RTD, locks the RTD oscillation to this signal, which in certain circumstance may reduce the phase noise of the RTD carrier, as discussed later. To distinguish between the carrier frequency of the AWG from the 
oscillation frequency (carrier) of the RTD, we call the former "subcarrier", which is the one that carries the baseband digital modulation.

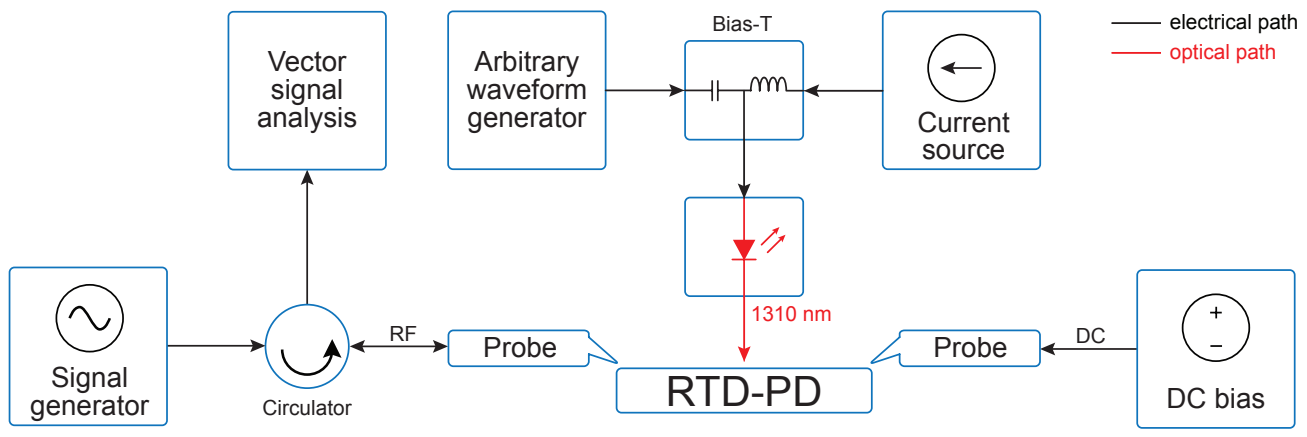

Figure 2. Experimental setup for optical modulation.

Figure 2 shows the experimental setup used for the transmission of advanced modulation formats, when using optical modulation. The setup is similar to the electrical modulation case, apart from the use of a laser diode that is directly modulated by the AWG signal, and whose fibre output is injected into the RTD-PD, through the alignment of the optical fibre on top of the RTD-PD optical window.

\section{EXPERIMENTAL RESULTS}

Figure 3 shows the spectrum of the electrical RTD carrier in the real-time spectrum analyser (RTSA) without injection locking (left) and with injection locking (right). To achieve RTD oscillation locking the signal generator power was set to $-15 \mathrm{dBm}$ and the frequency to $14.846 \mathrm{GHz}$, which are the same values used in the following results. Here we can observe the RTD carrier spectrum broadening due to the phase noise when unlocked, while when injection locked the phase noise is significantly reduced.
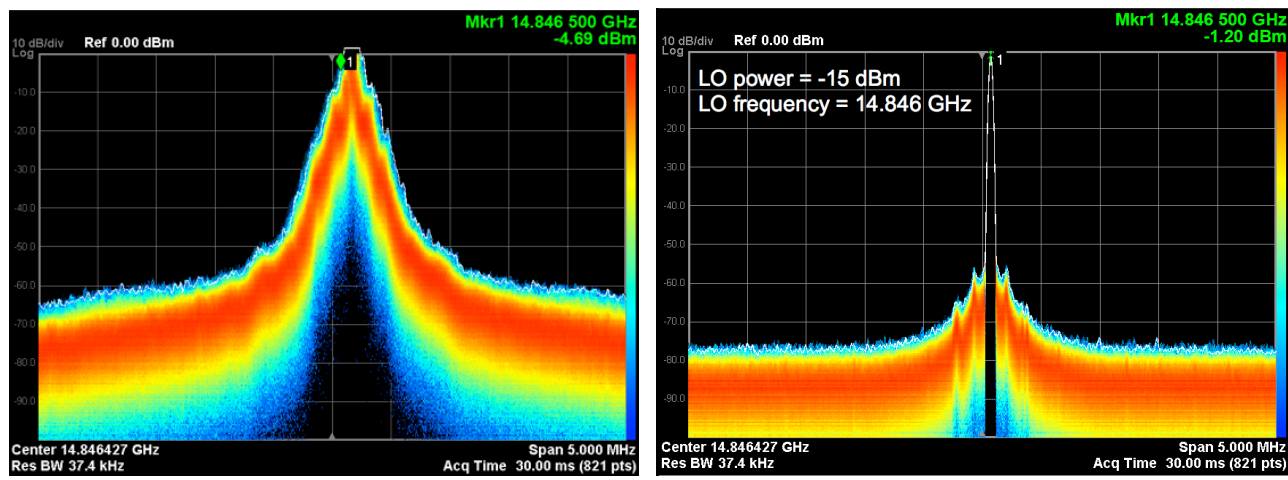

Figure 3. RTD carrier phase noise unlocked (left) and injection locked (right).

Using the setups described in Figure 1 and Figure 2, the response of the RTD to a subcarrier digital modulated signal is assessed experimentally.

Figure 4 shows the subcarrier channel power imposed on the RTD oscillation frequency, as a function of the subcarrier frequency obtained by transmitting 100 Mbaud QPSK signals generated by the AWG, for both electrical and optical modulation, transmitted with -11 and $-4 \mathrm{dBm}$ of power, respectively. This result shows a filtered frequency response (due to the moving average effect of the QPSK signal bandwidth), where the variations in amplitude with frequency lead to a spectral shaping of the subcarrier modulation therefore reducing the transmission performance. However, this can be corrected by applying an equalization filter to the transmitted signals. Moreover, it can be observed that the results with electrical modulation show much better performance than those with optical modulation. This is due to the lower amplitude of the RTD-PD modulated signal when using optical modulation, which is a result of the losses of the optical signal, namely the laser diode slope efficiency, the optical fibre coupling efficiency to the RTD-PD optical window, and the RTD-PD responsivity. These results are in agreement with the measured S21 with frequency offset (Figure 5). 


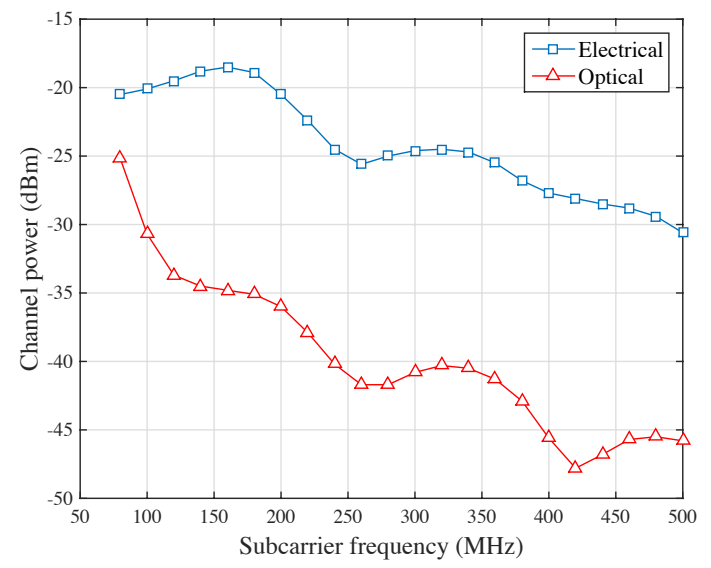

Figure 4. Channel power versus subcarrier frequency.

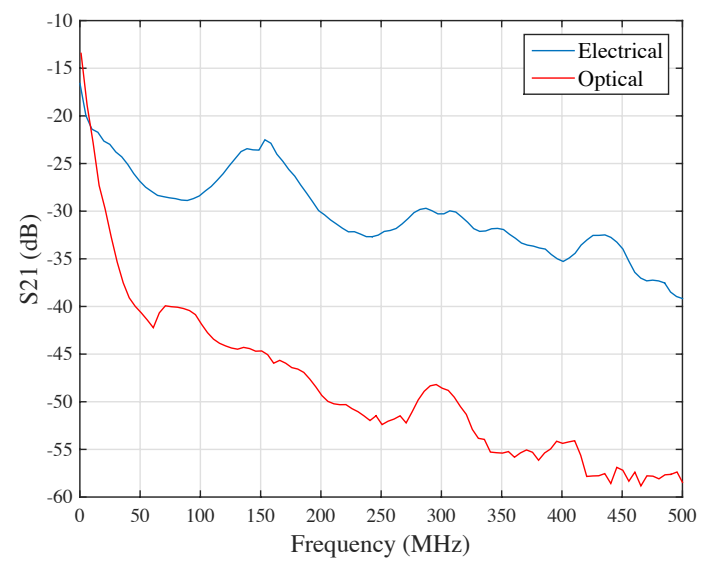

Figure 5. Measured S21.

Figure 6 shows the obtained experimental results in terms of the Signal to Noise Ratio (SNR), or equivalently, Modulation Error Ratio (MER), as a function of the subcarrier frequency used when modulated by the complex signal. In this experiment the AWG was generating QPSK signals with a symbol rate of $100 \mathrm{Mbaud}$. Also, the demodulated signals were equalized using the equalization filter available in the VSA software, which applies an adaptive filter to the measurement. One can conclude that the RTD-PD phase noise is only markedly affecting the results for subcarrier frequencies below approximately $250 \mathrm{MHz}$, since above this frequency, locking the RTD-PD oscillation does not provide a significant advantage. In this sense, the performance is limited by the phase noise for low subcarrier frequencies and limited by the signal amplitude for higher subcarrier frequencies.

Table 1 shows the transmitted signal constellations for different subcarrier frequencies and baud-rates. The first column shows the constellations transmitted electrically at $180 \mathrm{MHz}$, where the phase noise (visible in the constellation rotation) is completely compensated by injection locking. The second column shows the optical case at $120 \mathrm{MHz}$. Here we observe a similar result but with increased noise which leads to a lower SNR.

Figure 7 shows the SNR as a function of the symbol rate, obtained with a subcarrier frequency of $200 \mathrm{MHz}$ and both QPSK and 64-QAM modulation. We verify that without injection locking, the lower symbol rate performance, i.e. below around $60 \mathrm{MBaud}$, is reduced due to phase noise. This can be justified by the fact that lower symbol rate symbols are transmitted over a longer period of time and therefore experience additional phase noise [5]. Last column of

Table 1 shows the transmitted signal constellations with optical modulation at $200 \mathrm{MHz}$ with $10 \mathrm{MBaud}$ symbol rate. Here the phase noise when unlocked is more evident due to the lower symbol rate. For comparison, we also present in Figure 7 the SNR obtained with electrical modulation when transmitting 64-QAM signals with 20 and $100 \mathrm{MBaud}$ (also at $200 \mathrm{MHz}$ ), showing a performance penalty of around 2-4 dB.

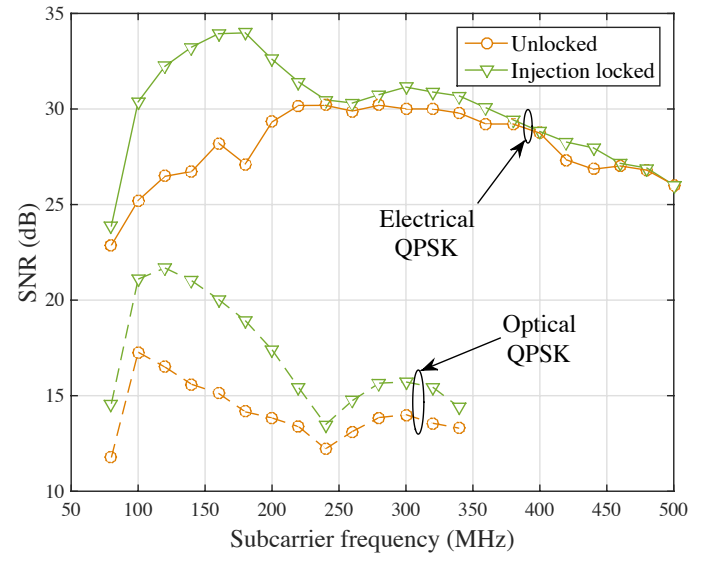

Figure 6. SNR versus subcarrier frequency obtained with a 100 MBaud QPSK subcarrier.

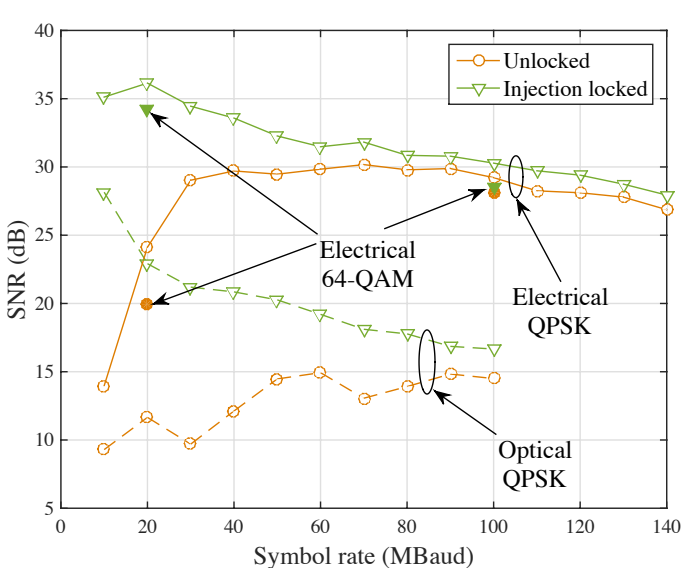

Figure 7. SNR versus symbol rate obtained with a 200 $M H z Q P S K$ subcarrier frequency and 64-QAM obtained with 20 and 100 MBaud. 
Table 1. Transmitted QPSK constellations

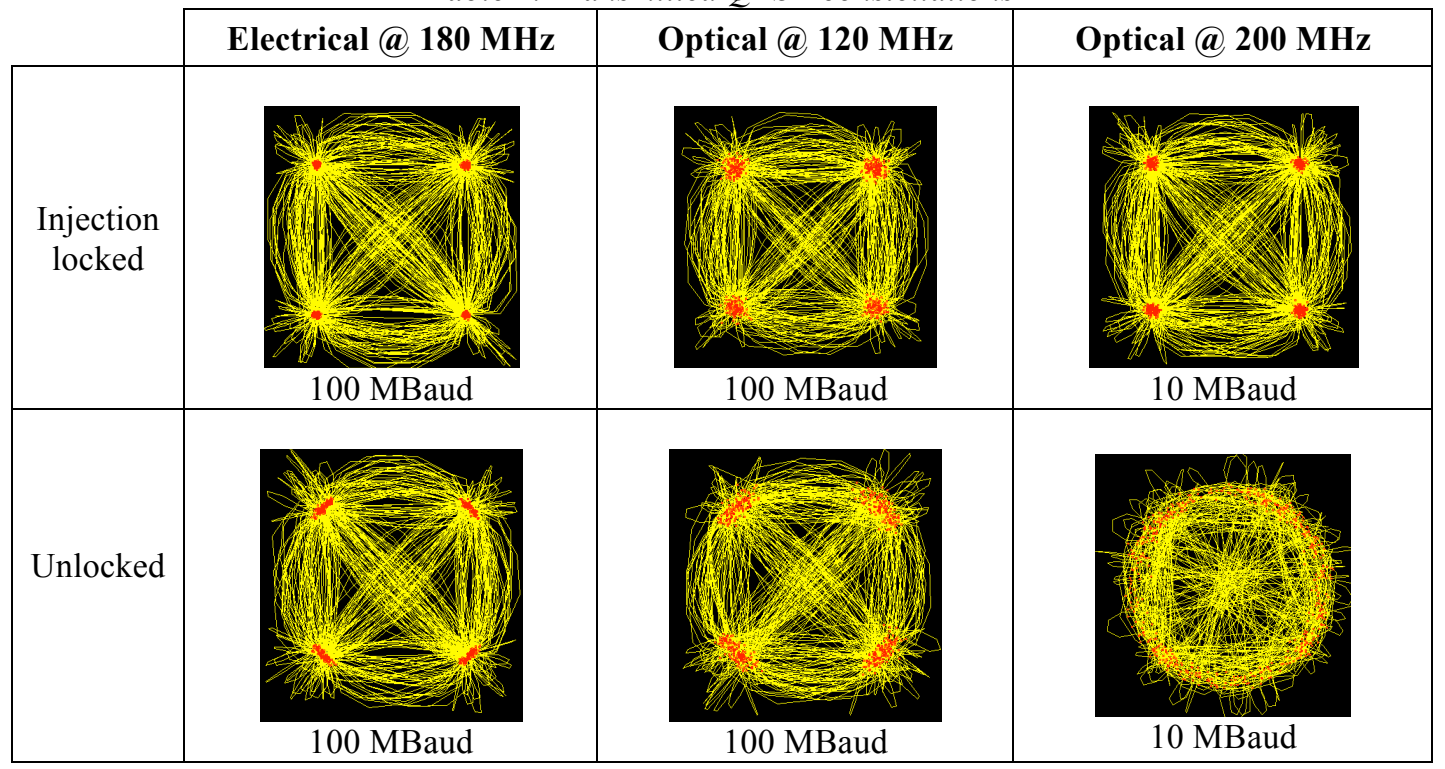

\section{CONCLUSIONS}

This paper comprises experimental evaluation and comparison of RTD-PDs transmission performance, using electrical and optical modulation, while employing, for the first time, advanced modulations formats. We verified that phase noise degrades system performance for subcarrier frequencies below $250 \mathrm{MHz}$ and for symbol rates below 60 MBaud. Although we did not quantify the phase noise, these preliminary experimental results show that phase noise is a limiting factor and in a near future we plan to experimentally measure the phase noise to supplement these results. Additionally, we can conclude that injection locking of the RTD with an external electrical signal between $100 \mathrm{MHz}$ and $200 \mathrm{MHz}$, provides a performance improvement in SNR of 3 to $5 \mathrm{~dB}$. Finally, electrical modulation shows better performance than optical modulation due to the losses associated with the optical signal and optoelectronic conversions.

\section{ACKNOWLEDGEMENTS}

The authors would like to thank to Edward Wasige, Jue Wang, Abdullah Al-Khalidi, Anthony Kelly and Scott Watson at the University of Glasgow for providing the RTD oscillator used in this work and José Figueiredo at the University of Lisbon for fruitful discussions.

This work was supported by the iBROW project (grant agreement no. 645369) as well as the TERAPOD project (grant agreement no. 761579), both supported by the European Commission under the Horizon 2020 Programme, and by FCT (Fundação para a Ciência e a Tecnologia, Portugal), through the research grant $\mathrm{PD} / \mathrm{BD} / 113821 / 2015$.

\section{REFERENCES}

[1] B. Romeira, L. Pessoa, H. M. Salgado, C. N. Ironside, and J. M. L. Figueiredo, Photo-detectors integrated with resonant tunneling diodes, Sensors, vol. 13 (7), pp. 9464-9482, Julho 2013.

[2] T. S. Moise,Y.-C. Kao, C. L. Goldsmith, C. L. Schow, and J. C. Campbell, High-Speed ResonantTunneling Photodetectors with Low-Switching Energy, IEEE Phot. Techn. Letters, 9, p. 803-805 (1997).

[3] J. Figueiredo, B. Romeira, T. Slight, C. Ironside, Advances in optical and photonic devices, InTech2010)

[4] J. Tavares, L. Pessoa, J. Figueiredo, and H. Salgado, "Analysis of resonant tunnelling diode oscillators under optical modulation," in Transparent Optical Networks (ICTON), 2017 19th International Conference on, 2017, pp. 1-4: IEEE.

[5] M. R. Khanzadi, D. Kuylenstierna, A. Panahi, T. Eriksson, and H. Zirath, "Calculation of the performance of communication systems from measured oscillator phase noise," IEEE Transactions on Circuits and Systems I: Regular Papers, vol. 61, no. 5, pp. 1553-1565, 2014. 